\title{
FRACTURE TOUGHNESS EVALUATION OF A Ni 2 MnGa ALLOY THROUGH MICRO INDENTATION UNDER MAGNETO-MECHANICAL LOADING
}

\author{
GOANŢ Viorel \\ Prof. Dr. Eng. \\ Department of Mechanical Engineering, Mechatronics and Robotics, „Gheorghe Asachi” Technical University of \\ Iaşi, Romania \\ CIOCANEL Constantin \\ Assoc. Prof. Dr. Eng. \\ Department of Mechanical Engineering, Northern Arizona University, AZ, USA
}

\begin{abstract}
Ni}_{2} \mathrm{MnGa}$ is a ferromagnetic alloy that exhibits the shape memory effect either induced by an externally applied magnetic field or mechanical stress. Due to the former, the alloy is commonly called magnetic shape memory alloy or MSMA. The microstructure of the MSMA consists of tetragonal martensite variants (three in the most general case) that are characterized by a magnetization vector which is aligned with the short side of the tetragonal unit cell. Exposing the MSMA to a magnetic field causes the magnetization vector to rotate and align with the external field, eventually leading to variant reorientation. The variant reorientation is observed macroscopically in the form of recoverable strain of up to $6 \%[1,2]$. As the magnetic field induced reorientation happens instantaneously [1, 3], MSMAs are suitable for fast actuation, sensing, or power harvesting applications. However, actuation applications are limited by the maximum actuation stress of the material that is about 3.5MPa at approximately 2 to $3 \%$ reorientation strain. During MSMA fatigue magneto-mechanical characterization studies [4, 5] it was observed that cracks nucleate and grow on the surface of material samples, after a relatively small number of cycles, leading to loss in material performance. This triggered the need for understanding the mechanisms that govern crack nucleation and growth in MSMAs, as well as the nature of the material, i.e. ductile or brittle. The experimental study reported in this paper was carried out to determine material's fracture toughness, the predominant crack growth directions, and the orientation of the cracks relative to the mechanical loading direction and to the material's microstructure. A fixture has been developed to allow Vickers micro indentation of $3 \mathrm{~mm}$ by $3 \mathrm{~mm}$ by $20 \mathrm{~mm} \mathrm{Ni} \mathrm{i}_{2} \mathrm{MnGa}$ samples exposed to different levels of magnetic field and/or mechanical stress. Using the measured characteristics of the impression generated during micro indentation, the lengths of propagated cracks, and appropriate equations (introduced in the paper), and the fracture toughness was evaluated as a function of the magneto-mechanical loading experienced by the material. The influence of the magneto-mechanical loading on the growth of already nucleated cracks has also been evaluated.
\end{abstract}

Key words: ferromagnetic alloy, fracture mechanics, martensite variants

\section{Brief intro to magnetic shape memory alloys}

Magnetic shape memory alloys (MSMAs) can exhibit either magnetic field or stress induced martensite reorientation. The most common MSMA alloy is $\mathrm{Ni}_{2} \mathrm{MnGa}$ which is engineered to have a twinmartensite microstructure. The martensitic phase consists of tetragonal unit cells, as shown in Figure 1, which are characterized by a short side (c) and a long side (a), with the former also corresponding to the direction of the easy axis of magnetization (EA) [6,7]. The magnetic easy axis (denoted EA in Figure 1) of the internal magnetization vectors is approximately aligned with the short side of the unit cell, as shown in Figure 1. When the material is placed in a magnetic field, the magnetization vectors tend to align with the external field in order to minimize the magnetic potential energy of the material. This is achieved by rotating the internal magnetization vectors away from the magnetic easy axis and towards the external field and/or by reorienting from one variant to another. Alternatively, when a compressive stress is applied to the material, the short axis of the unit cell tends to align with the direction of the compressive stress. 
ACTA UIVERSITATIS CIBINIENSIS - TECHNICAL SERIES

Vol. LXIX

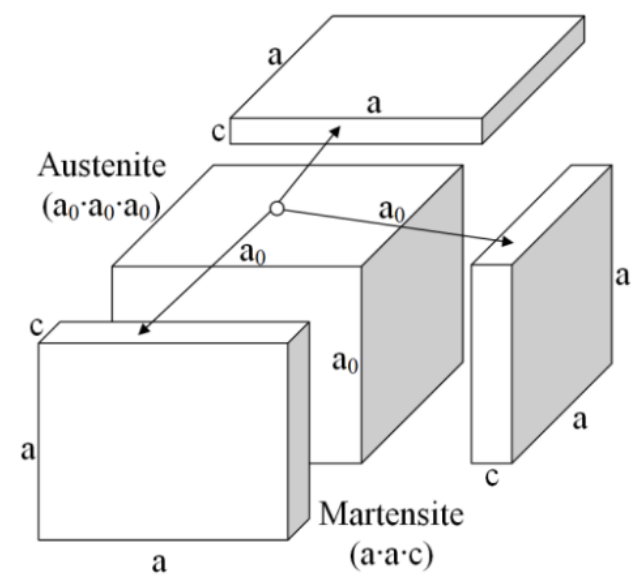

Figure 1: The three possible tetragonal martensite variants derived from the austenite parent phase

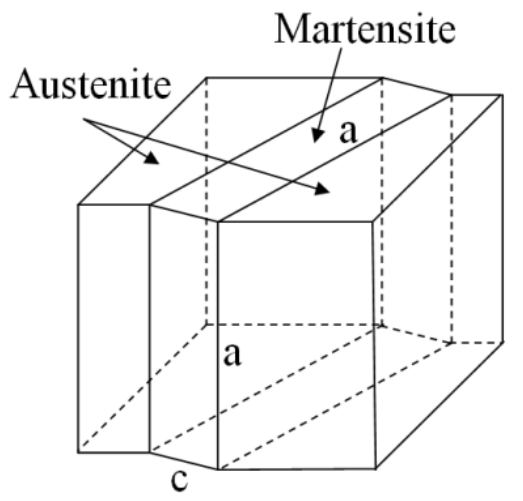

Figure 2: A schematic showing tetragonal variants of martensite present in the material and their coexistence along the twinning plane

The macroscopically observed effect of the variant reorientation, for the alloy investigated in this work, is strain reaching approximately 6\%. This strain can then be recovered, as shown in Figure 3.a, by applying an axial compressive stress on the material while keeping the bias magnetic field on. The field induced variant reorientation occurs very fast (in the order of milliseconds) and can be used to actuate loads that develop stresses as large as $3.5 \mathrm{MPa}$ (note that Figure 3.a illustrates the stress required to recover the reorientation strain, not the actuation stress). Beyond this stress level, the variant reorientation cannot be induced by the magnetic field, irrespective of its magnitude. These characteristics make the material suitable for a wide range of applications, including actuators, sensors and power harvesters. In such applications, the material needs to complete millions of magneto-mechanical cycles which can yield to material fatigue.

During cyclic magneto-mechanical loading specific to power harvesting/sensing applications, i.e. loading with magnetic field applied normal to the length of the sample and with a compressive axial stress applied along the length of the sample (normal to the field direction), material samples may developed cracks as illustrated in Figure 3.b.

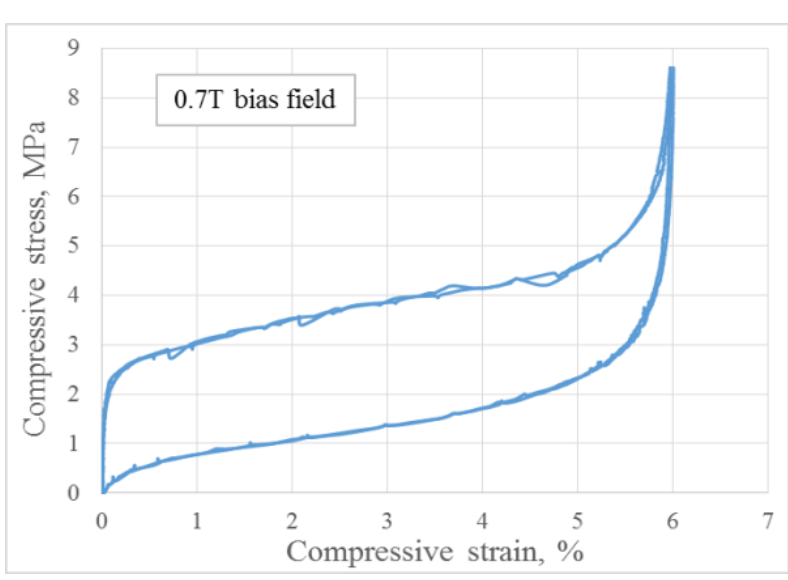

a)
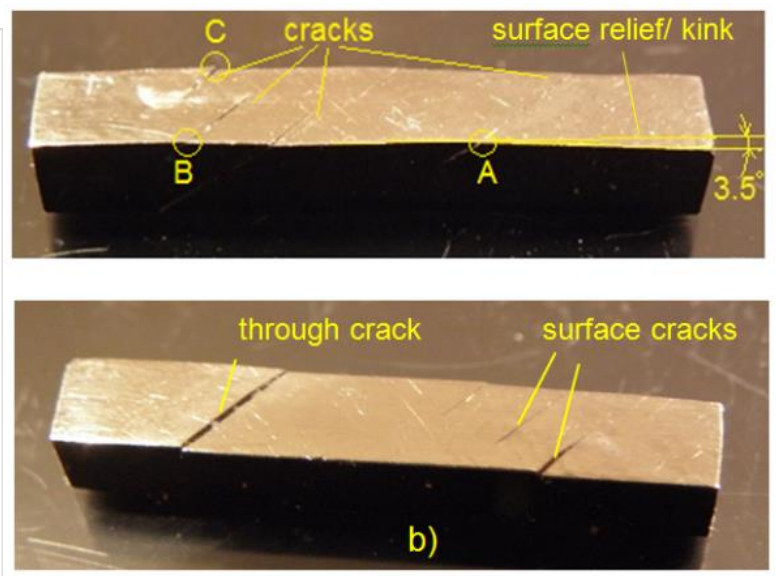

b)

Figure 3: a) Macroscopic response of the material exposed to transverse bias magnetic field of $0.7 \mathrm{~T}$ and axial compressive stress. b) Samples that developed surface and through cracks during magneto-mechanical loading. 
ACTA UIVERSITATIS CIBINIENSIS - TECHNICAL SERIES

Vol. LXIX 2017

Fatigue studies that investigated either the effect of magnetic or mechanical loading on material's performance have been reported in $[4,5,8]$ and provided valuable insight on the long term behavior of the material. However, there are still a few questions left unanswered, particularly regarding the effect of combined magneto-mechanical loading on the long term behavior of the material. Furthermore, the mechanisms that govern crack propagation and growth in these materials is not fully understood. Related to that, and still unavailable, is the fracture toughness of the material. Consequently, this paper attempts to provide insight on both, crack growth mechanisms as well as fracture toughness for the material. This information will assist design engineers in making informed decisions on the feasibility of the material for a wide range of applications.

\section{Fracture Toughness Evaluation for a $\mathrm{Ni}_{2} \mathrm{MnGa}$ Alloy}

Understanding fracture magneto-mechanics of magnetic shape memory alloys, and in particular of the $\mathrm{Ni}_{2} \mathrm{MnGa}$ alloy, is critical for realizing the full potential of these materials in practical applications.

Having comprehensive information on material's behavior and its properties relevant to static and dynamic applications allows engineers to design more efficient and more reliable $\mathrm{Ni}_{2} \mathrm{MnGa}$ based actuators and sensors. Given that these materials are used almost exclusively in compression, there is no tensile test data available to allow one to evaluate the ductile or brittle character of the material from the material characteristic curve. One could argue, based on the amount of reorientation strain exhibited by the material that it is ductile, but observations of cracks developing in the material during magneto-mechanical loading, along with the nature of the cracks, suggest that its character is brittle or quasi-brittle. The brittle character is likely enhanced by the lamellar structure of the alloy, as cracks appear to nucleate and grow mainly along twin boundaries (see Figures 3.b and 4.a). However, cracks have been observed to grow across twins as well, as showed in Figure 4.b, supporting the hypothesis on the brittle character of the material and making a stronger case for the work reported here.

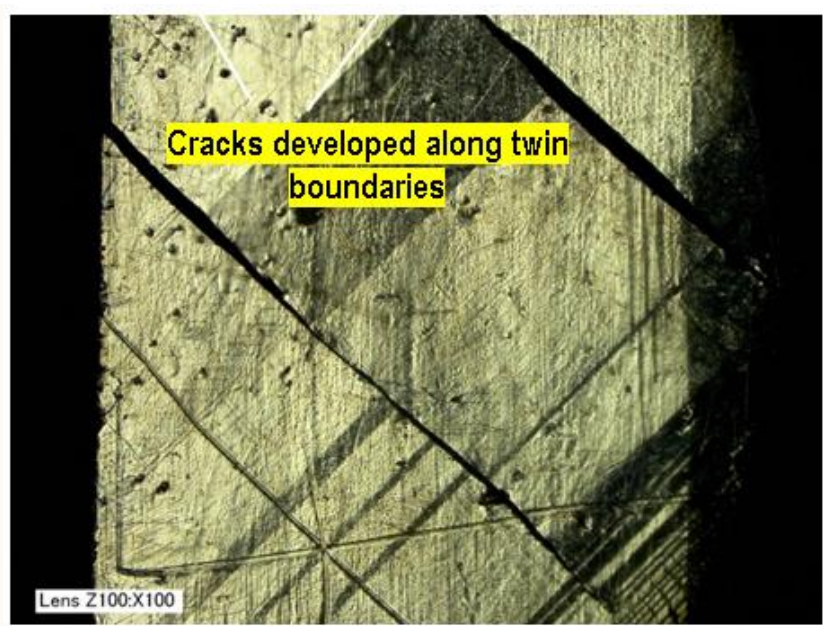

a)

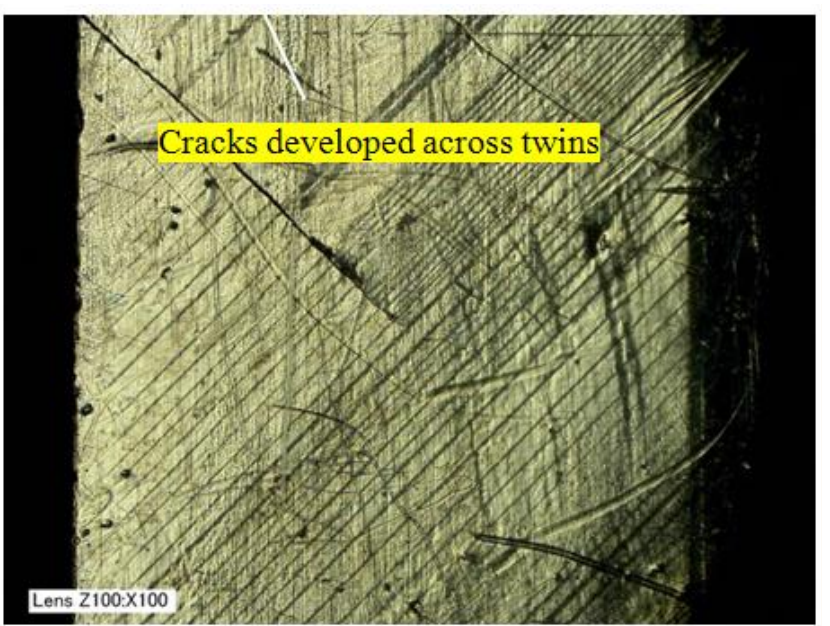

b)

Figure 4: Images illustrating a) cracks developed along twin boundaries and b) cracks developed across twins.

\section{Approach}

In order to evaluate MSMAs fracture toughness and to dive deeper into understanding the crack growth mechanisms in these alloy, we deployed a combined experimental approach augmented with established analytical computations. Each component of this approach is detailed in the following paragraphs. 


\section{ACTA UIVERSITATIS CIBINIENSIS - TECHNICAL SERIES}

Vol. LXIX 2017

\subsection{Experimental approach}

In order to determine the fracture toughness of the MSMA, we performed Vickers micro indentation hardness tests on it. Micro-indentation tests can be deployed to evaluate material's fracture toughness because during indentation cracks are expected to nucleate at the edges of the indenter tip and subsequently grow in directions that are dependent on the material microstructure, which is indicative of the brittle nature of the material. Furthermore, using the characteristics of the cracks (i.e. crack length at the end of the micro indentation) and the geometry of the impression (i.e. its projected size on the plane of the material surface where the indentation was performed), one can calculate the fracture toughness, $\mathrm{K}_{\mathrm{Ic}}$, for the material.

The work reported here was carried out on a two-variant $\mathrm{Ni}_{2} \mathrm{MnGa}$ alloy (specifically $\quad \mathrm{Ni}_{50} \mathrm{Mn}_{28.5} \mathrm{Ga}_{21.5}$ ) prismatic sample, with sides $2 \mathrm{~mm}$ by $2 \mathrm{~mm}$ by $20 \mathrm{~mm}$ that was loaded magneto-mechanically with an in-house developed device shown in Figure 5. This device exposes the MSMA sample to a range of magneto-mechanical loading conditions, by using permanent magnets to generate a field of $0.6 \mathrm{~T}$ at the MSMA sample surface and a micrometer screw to apply an axial load of up to $70 \mathrm{~N}$ on the sample. The axial load and magnetic field directions are mutually perpendicular. The axial load provided by the micrometer screw, along with the capability of the screw to measure the MSMA's displacement, allows for micro-indentations

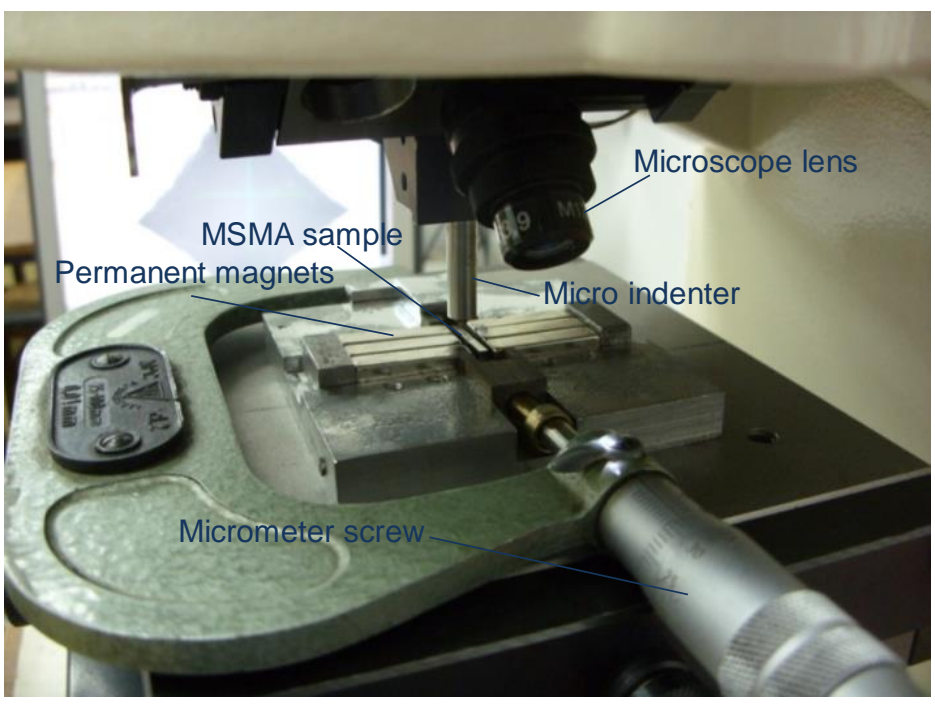

Figure 5: Magneto-mechanical loading jig installed on the table of a Emcotest EMC10 micro hardness device. to be performed at various strain levels on the MSMA sample. This magneto-mechanical loading device is installed on the table of a micro hardness device, Emcotest EMC10, where micro indentations of the sample are being carried out. The entire setup allows for the visualization and measurement of the micro-indentation impressions and of the length of the propagated cracks.

A schematic of the fine two-variants structure of the tested material is shown in Figure 6, along with the directions of the magnetic field (B) and compressive mechanical stress $\left(\sigma_{\mathrm{xx}}\right)$ for the case when the microindentation was performed on Surface 2. Micro-indentations were performed with the indenter in two orientations, denoted in the figure as 0 and 45 degrees, based on the angle made by the side of the impression identified in the figure with the twin boundary. Similarly, indentations at 0 and 45 degrees were also performed on Surface 1. 
Vol. LXIX

Figure 6: Schematic of the "fine twin-variant microstructure" of the tested $\mathrm{Ni}_{2} \mathrm{MnGa}$ sample, indicating the mechanical and magnetic field loading directions as well as the orientations of the micro-indentations relative to the twin boundary on Surface 2 of the sample.

Microindentations have been performed, under five magneto-mechanical loading conditions, on both surfaces (Surface 1, which exhibits the surface relief showed in Figure 3.b, and Surface 2), as follows:

1. the sample in the absence of magnetic field and axial loading (i.e. the sample was all in variant 2);

2. the sample was kept compressed and a 0.6T field was applied normal to surface 1;

3 . in the absence of a compressive stress, the sample was exposed to 0.6T magnetic field applied normal to surface 1(i.e. sample fully expanded);

4. starting from the fully expanded state, the sample was compressed $2.25 \%$ while exposed to $0.6 \mathrm{~T}$ magnetic field applied normal to surface 1 ;

5. starting from a $2.25 \%$ compressive strain, in the presence of $0.6 \mathrm{~T}$ magnetic field applied normal to surface 1 , the sample was compressed an additional $2.25 \%$ strain, to a maximum of $4.5 \%$ compressive strain.

The indentation load was $294.3 \mathrm{~N}$ and the indenter used was a square based pyramid with a $136^{0}$ dihedral angle.

\subsection{Analytical approach complementing the experimentation}

The Vickers micro-indentation method is commonly deployed to facilitate the evaluation of a material's fracture toughness in conjunction with the micro-crack approach [9].In this approach, an empirical expression is derived for the fracture toughness $\mathrm{K}_{\mathrm{Ic}}$ based on the dimensions of the impression and on the size of the cracks propagated during micro-indentation. This empirical expression for $\mathrm{K}_{\mathrm{Ic}}$ often contains calibration factors that must be evaluated through means other than the micro-crack approach. However, in order to rely on Vickers micro-indentation data for fracture toughness calculations, one has to derive the expressions for the maximum stress developed near the impression after the indentation, as

$$
K_{I C}=\sigma \sqrt{\pi \cdot a}
$$

The maximum stress can be determined assuming that a crack propagated during micro-indentation has a half-penny shape as shown schematically in Figure 7.a. Performing a finite element analysis on a material volume that experienced a micro-indentation, it is found that the maximum stress that causes crack propagation, i.e. the hoop stress, occurs at the tip of the indenter (see the red arrows shown in Figure 7.b). 
ACTA UIVERSITATIS CIBINIENSIS - TECHNICAL SERIES

Vol. LXIX 2017

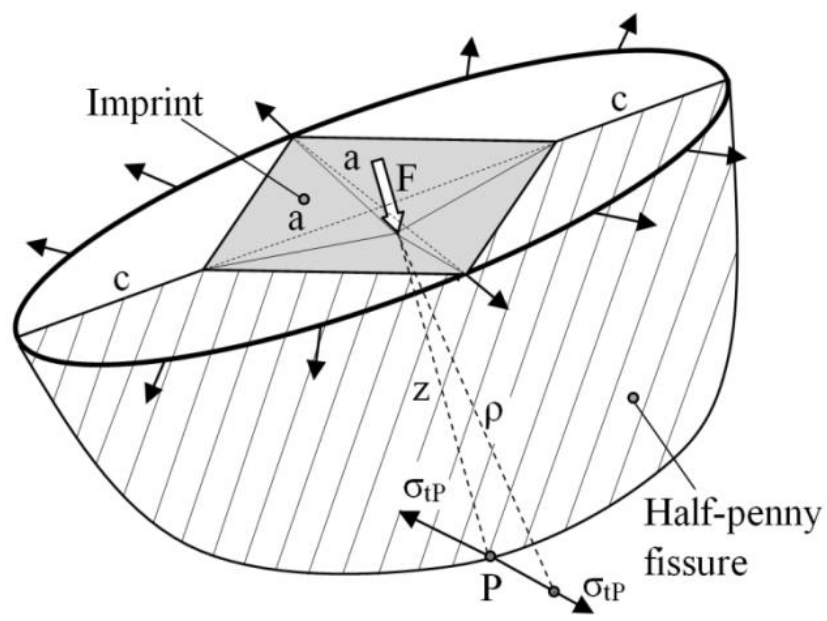

a)

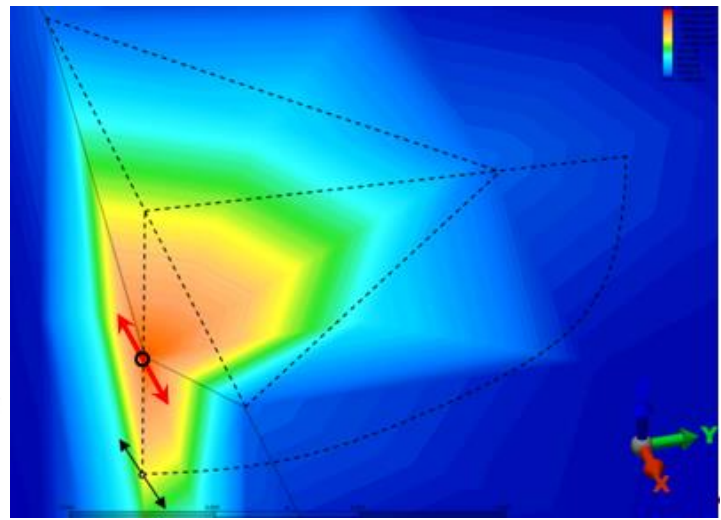

b)

Figure 7: a) Assumed geometry of the micro crack for stress analysis purposes. b) FEA results indicating the stress distribution around the indentation.

Boussinesq [10] proposed the expression for the hoop stress, $\sigma_{t}$, of the form showed in Equation (2), assuming the indentation to have been performed on a planar surface, with a Vickers indenter, through the application of a load $\mathrm{F}$.

$\sigma_{t}=\frac{F}{2 \pi}(1-2 v)\left[\frac{z}{\rho^{3}}-\frac{1}{\rho(\rho+z)}\right]$

In Equation 2, $\mathrm{z}$ is the depth of the micro crack measured from the tip of the indenter, $\rho$ is the coordinate of a point in the vicinity of the edge of the micro crack, measured also from the tip of the crack, and $v$ is material's Poisson ratio.

Given that the maximum hoop stress occurs at point $P(\rho=z)$ shown in Figure 7.a, and based on the geometrical relationships between $\mathrm{z}$, a (half of the diagonal of the impression) and $\mathrm{c}$ (half of the length of the propagated crack), the expression for the hoop stress becomes:

$\sigma_{t P}=\sigma_{I c}=\frac{(1-2 v) F}{4 \pi a^{2}} \ln \frac{a^{2}+c^{2}}{c^{2}}$

Substituting this stress expression in the equation defining the fracture toughness yields:

$\mathrm{K}_{\mathrm{Ic}}^{1}=\frac{(1-2 v) \mathrm{F} \sqrt{\mathrm{a} \cdot 10^{-6}}}{2 \sqrt{2 \pi}\left(\mathrm{a} \cdot 10^{-3}\right)^{2}} \ln \frac{\mathrm{a}^{2}+\mathrm{c}^{2}}{\mathrm{c}^{2}}$

where the units for $K_{I c}$ are $\mathrm{MPa} \cdot \mathrm{m}^{1 / 2}$ provided that the units for $F, a$ and $c$ are Newtons $(\mathrm{N})$ and micrometers $(\mu \mathrm{m})$, respectively.

Alternatively, fracture toughness may be calculated using Equation (5) 
ACTA UIVERSITATIS CIBINIENSIS - TECHNICAL SERIES

Vol. LXIX 2017

$$
\mathrm{K}_{\mathrm{Ic}}^{2}=(0.016)\left(\frac{\mathrm{E}}{\mathrm{H}}\right)^{\frac{1}{2}}\left(\frac{\mathrm{F}}{\mathrm{c}^{\frac{3}{2}}}\right)
$$

that accounts for the modulus of elasticity and Vickers hardness of the material, in addition to the crack geometry,or with Equation (6) that accounts for material's Vickers hardness, crack geometry and impression geometry:

$\mathrm{K}_{\mathrm{Ic}}^{3}=(0.16)\left(\frac{\mathrm{c}}{\mathrm{a}}\right)^{-\frac{3}{2}}\left(\mathrm{Ha}^{\frac{1}{2}}\right)$.

The value of the Poisson ratio used in Equation (4) was $v=0.3$, and the modulus of elasticity of the material from Equation (5) was determined to be $\mathrm{E}=32.213 \mathrm{GPa}$. The latter was determined through a nanoindentation test performed on the MSMA sample according to ISO 14577-1. Also, the material hardness required in Equations (5) and (6) was calculated, in units of Vickers, HV, using Equation (7)

$\mathrm{H}=\left[(0.1891 \cdot 294.3) /\left(4 \mathrm{a}_{\mathrm{n}}^{2}\right)\right] \cdot 10^{6}$

in which the half diagonal value of the nano-impression, $a_{n}$, was determined from the same nano-indentation test performed on the MSMA sample. In Equation (7), the units for $a_{n}$ are micrometers $(\mu \mathrm{m})$. The nanohardness test was performed under a magnetic field of 0.6T applied normal to Surface 1 (see Figure 6). Nanoindentations have been carried out for all the magneto-mechanical cases reported above, and Young modulus was calculated for each loading case.

The fracture toughness, $K_{I C}$, was calculated using Equations (4) through (6), and subsequently a decision was made on which equation yields the most reliable value to be reported for the fracture toughness of the material.

\section{Results}

For each of the magneto-mechanical loading conditions described above, twelve micro indentations have been performed on each surface of the sample (i.e. on Surface 1 and Surface 2) (see Figure 6), and a weighted average crack length was calculated based on each set of twelve measurements; this average crack length was then used to calculate material's fracture toughness using Equations (4) through (6). When the extreme value of the crack length was larger by $15 \%$ than the weighted average, it was discarded from the calculations. A sample measurement of the crack length, the diagonals of the impressions, and of other features of the impressions is illustrated in Figure 8. For each impression, the $2 \mathrm{a}_{1}$ and $2 \mathrm{a}_{2}$ diagonals (see Figure 8.a) have been measured and half of the average of these two values was used to calculate $K_{I C}$. The crack length $c$ was determined by measuring the crack length outside the impression, i.e. measuring dimension e showed in Figure 8.b, and adding to it the distance from the edge of the impression to the center of the impression, i.e. $a / \sqrt{2}$ (showed in Figure 8.a). It has to be noted that the lengths of all cracks that propagated during indentation, in all five cases considered, were measured and the largest crack length in each test case was then used to calculate the fracture toughness associated with that loading case. When the magnetic field was present, it was applied only normal to Surface 1, as only in this orientation causes variant reorientation. 
ACTA UIVERSITATIS CIBINIENSIS - TECHNICAL SERIES

Vol. LXIX 2017

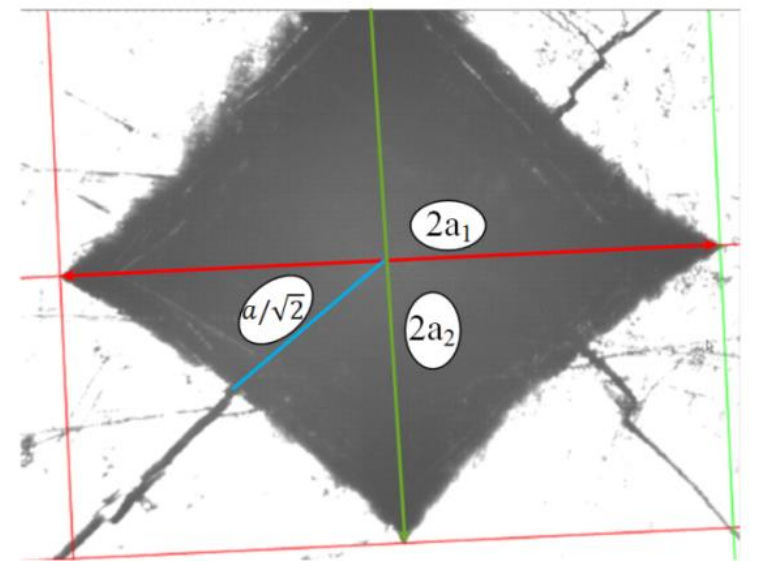

a)

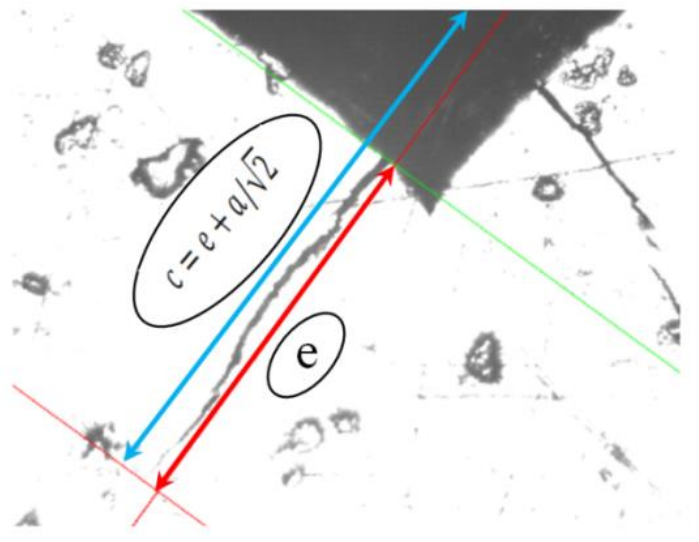

b)

Figure 8: a) A sample impression and propagated cracks during the micro-indentation of the $\mathrm{Ni}_{2} \mathrm{MnGa}$ sample; b) crack length measurement approach.

Figures 9 and 10 summarize the values calculated for fracture toughness using the three equations introduced earlier, for each loading case, sample orientation relative to the indenter, and surface of indentation. Figure 9 summarizes the results obtained from the indentations carried out on Surface 1, while Figure 10 summarizes the results for the indentations carried out on Surface 2 (the loading cases 1 through 5 have been introduced in section 3.1 above).

It has to be noted that the three equations used to calculate $\mathrm{K}_{\text {Ic }}$ have one parameter in common, the crack length $\boldsymbol{c}$, suggesting that the difference in the computed fracture toughness values must be attributed to the contributions of the other parameters appearing in these equations, i.e. the length a of half of the diagonal of the impression, the applied force F, the Vickers hardness HV30 and Young's modulus E. The results indicate that not only the magneto-mechanical loading but also the orientation of the indenter relative to twin boundaries influences the calculated values for $\mathrm{K}_{\mathrm{Ic}}$. The results showed in Figure 9.a suggest that application of the magnetic field on the fully compressed sample (i.e. loading case 2) tends to slightly increase the fracture toughness, but as soon as the material is allowed to reorient (i.e. loading cases 3 and 4), the fracture toughness decreases. The latter tends to suggest that keeping the magnetic field constant at $0.6 \mathrm{~T}$ but reducing the compressive stress facilitates crack propagation in the material, hence lower $\mathrm{K}_{\mathrm{Ic}}$ values. Once the material is fully reoriented, the fracture toughness increases to levels comparable to that calculated for loading case 2. This makes sense as both cases, case 2 and case 5, are associated with the material being all in a one variant state, which are less conducive states for crack nucleation and growth. Figure 9.b that presents the fracture toughness values for the indenter rotated 45 degrees relative to the Figure 9.a orientation indicate a progressive increase in fracture toughness with the presence of the field and reduction in compressive stress. These results are unexpected as the removal of the compressive stress was expected to yield a decrease in fracture toughness values. Figure 10 summarizes the results for indentations on Surface 2 for the same two orientations of the indenter relative to the twin boundaries. Both, the results showed in Figure 10.a and those from Figure 10.b suggest that the presence of the magnetic field and the progressive decrease in compressive stress facilitate crack propagation, hence the lower fracture toughness values recorded for loading cases 4 and 5 in Figure 10.a and 3, 4 and 5 in Figure 10.b. Comparing the fracture toughness values determined on both surfaces reveals that Surface 2 yielded fracture toughness values almost twice the magnitude of those determined from Surface 1. This can be due to the fact that indentations on Surface 1 are not likely accompanied by local variant reorientation, which causes immediate crack nucleation and growth, leading to 


\section{ACTA UIVERSITATIS CIBINIENSIS - TECHNICAL SERIES}

Vol. LXIX 2017

higher crack lengths and lower fracture toughness values. The higher the compressive stress, the higher the fracture toughness, suggesting that the compressive stress is hampering crack nucleation and growth.

The fully elongated material exhibits higher fracture toughness when measured on Surface 1 and lower fracture toughness when measured on Surface 2. This is likely due to the fact that, in the latter, the indenter is acting in the direction that facilitates variants toppling under stress, as opposed to acting on Surface 1 which does not contain variants that would switch orientation (e.g. var. 3 into var. 1).

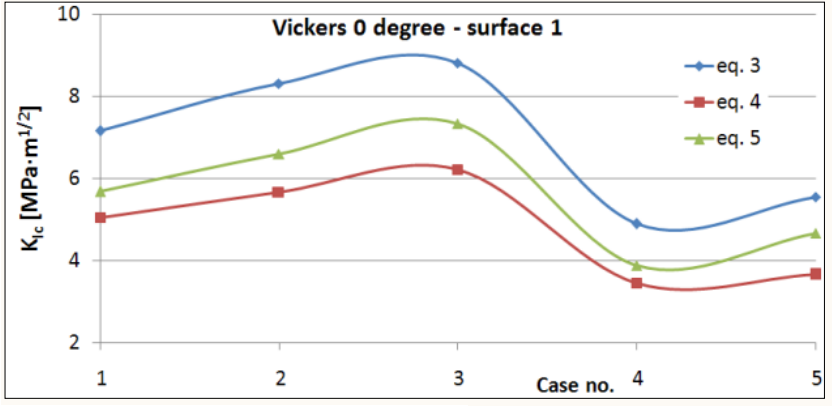

a)

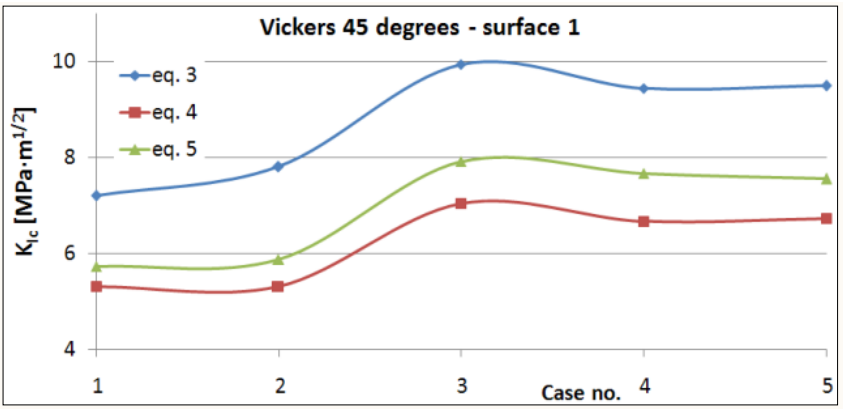

b)

Figure 9: Fracture toughness calculated based on indentation results for Surface 1

The analysis of Figures 9.a. and 9.b that present the indentation results in both orientations performed on Surface 1, indicates that:

- fracture toughness for case 2 is larger than surface toughness for case 1 (i.e. tests with constraint sample vs. free sample, keeping the magnetic field constant at $0.6 \mathrm{~T}$ ), and the increase in fracture toughness is larger for the 0 degree indentation than for the 45 degrees indentation:

$\Delta K_{I c(2-1), 0 \text { degree }, S 1}=0.89 \mathrm{MPa} \sqrt{\mathrm{m}} ; \quad \Delta K_{I c(2-1), 45 \text { degrees }, S 1}=0.25 \mathrm{MPa} \sqrt{\mathrm{m}}$

- when the axial load is removed but the magnetic field is still applied (i.e. case 3), the fracture toughness increases further, and a larger increase occurs in the 45 degrees test compared to the 0 degrees test:

$\Delta K_{I C(3-2), 0 \text { degree }, S 1}=0.59 \mathrm{MPa} \sqrt{m} ; \quad \Delta K_{I c(3-2), 45 \text { degrees }, S 1}=1.96 \mathrm{MPa} \sqrt{m}$

- fracture toughness decreases significantly with the sample compression to achieve a $2.25 \%$ strain, under magnetic field, at 0 degree indentation:

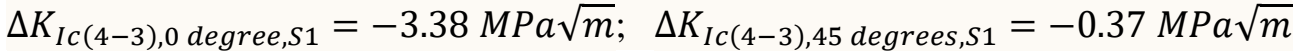

- fracture toughness remains low with further increase in the compressive stress (i.e. $4.5 \%$ strain) (case 5). An increase in fracture toughness is observed, relative to the previous test condition, only for the 0 degrees test; the 45 degrees tests yielded almost identical fracture toughness values:

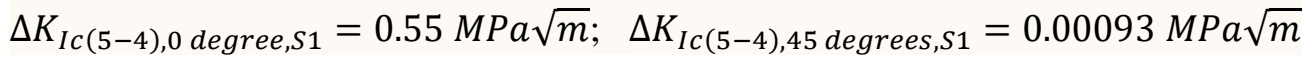


ACTA UIVERSITATIS CIBINIENSIS - TECHNICAL SERIES

Vol. LXIX 2017

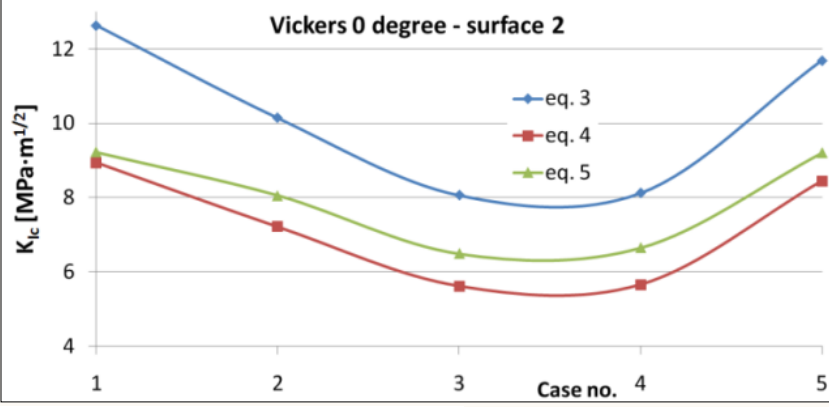

a)

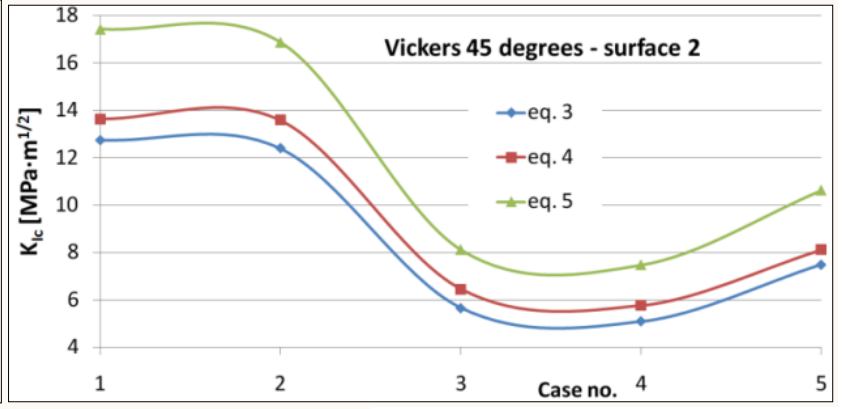

b)

Figure 10: Fracture toughness calculated based on indentation results for Surface 2.

Overall, the data suggests that the material has a brittle character (due to the relatively low values for $\mathrm{K}_{\mathrm{Ic}}$ ) and that the orientation of the indenter relative to the twin boundary affects the spread of the predictions between equations. If Equation (3) predicts a larger $\mathrm{K}_{\mathrm{Ic}}$ value for the indenter at 0 degrees with respect to the twin boundary, it predicts a lower value when the indenter is at 45 degrees relative to the twin boundary.

The analysis of Figures 10.a. and 10.b that present the indentation results in both orientations performed on Surface 1, indicates that:

- once the magnetic field is applied and the fracture toughness is maintained so that the length is maintained at the initial value, case 2, the fracture toughness decreases slightly from the initial moment, the decrease being greater for indentation at 0 degrees:

$\Delta K_{I c(2-1), 0 \text { degree }, S 2}=-1.79 \mathrm{MPa} \sqrt{\mathrm{m}} ; \quad \Delta K_{\text {Ic }(2-1), 45 \text { degrees }, S 2}=-0.32 \mathrm{MPa} \sqrt{\mathrm{m}}$

- fracture toughness decreases more with the reduction in compressive stress (case 3 ), while the magnetic field is still present. This is expected as the mobility of the variants increases and separation of the variants at their twin boundaries are more likely. A larger decrease in fracture toughness is recorded when the indentation was done at 45 degrees:

$\Delta K_{\text {Ic }(3-2), 0 \text { degree, } S 2}=-1.74 \mathrm{MPa} \sqrt{\mathrm{m}} ; \quad \Delta K_{\text {Ic }(3-2), 45 \text { degrees }, S 2}=-7.54 \mathrm{MPa} \sqrt{\mathrm{m}}$

- a small increase in the mechanical stress, to induce $2.25 \%$ reorientation strain, does not lead to an increase in fracture toughness (case 4);

- while keeping the field constant and increasing the stress to induce a $4.5 \%$ reorientation strain, it is observed that the fracture toughness increases for both indentation orientations, 0 and 45 degrees. Again, this increase is likely due to the compressive mechanical stress that compacts the material more, reducing the possibility of crack nucleation. In addition, the increase in fracture toughness is very consistent for both orientations, when compared to testing case 5, i.e.:

$\Delta K_{\text {Ic (5-4), } 0 \text { degree }, S 2}=2.96 \mathrm{MPa} \sqrt{\mathrm{m}} ; \quad \Delta K_{\text {Ic }(5-4), 45 \text { degrees }, S 2}=2.63 \mathrm{MPa} \sqrt{\mathrm{m}}$

In general, when evaluated on surface 1, fracture toughness is lower than when evaluated on surface 2:

$K_{I C M E D, S 2}=9.25 \mathrm{MPa} \sqrt{m} ; \quad K_{I C M E D, S 1}=6.55 \mathrm{MPa} \sqrt{m}$

\section{Conclusions}

The fracture toughness of a $\mathrm{Ni}_{2} \mathrm{MnGa}$ alloy has been investigated to provide engineers with a more complete set of properties for the material that will assist them to better design devices that rely on MSMAs for actuation and sensing. Knowledge of the fracture toughness characteristics of the alloy, particularly with 
respect to most possible magneto-mechanical loading conditions, is critical given the high cycle applications (i.e. actuation, sensing, and power harvesting) for which this material is commonly used.

In this paper, the fracture toughness of a $2 \mathrm{~mm}$ by $2 \mathrm{~mm}$ by $20 \mathrm{~mm} \mathrm{Ni2MnGa}$ sample was evaluated using a magneto-mechanical loading device designed in house to fit a Vickers 30 micro indentation test rig. The sample was indented, under five magneto-mechanical loading conditions, on two surfaces oriented at 90 degrees relative to each other, and with two orientations of the indenter, i.e. 0 and 45 degrees with respect to the twin boundaries. Using the geometry of the impression and the dimensions of the developed cracks, material's fracture toughness has been calculated with three different equations, with only one common parameter among all three, i.e. crack length c. The method deployed in this study is commonly called indentation fracture, (IF).

The calculated values for fracture toughness, in all tested conditions, suggest that the material has a brittle nature, and that the fracture toughness depends on the magneto-mechanical loading of the material.

The stress that causes crack nucleation is the hoop stress given by Equation (2) and showed in Figure 5. Application of a magnetic field causes the material to reorient, and depending on the ratio between the magnitudes of the field and the compressive stress, the material will switch from one state into the other very fast, almost exhibiting instability. Such a condition favors crack nucleation and growth in the material, and consequently the observed lower fracture toughness on Surface 2 when the field was initially applied on the material. It is to be noted that the compressive stress causes stresses in the vicinity of the crack that reduce the magnitude of the hoop stress that initiates and grows the crack. This explains the observed slight increase in $\mathrm{K}_{\mathrm{Ic}}$ after compression. Nonetheless, the fracture mode is dependent on the orientation of the lamellar structure of the material relative to the stresses acting on the material. The results suggest that not only the compressive stress but also the magnetic field (through variant reorientation) influence the crack propagation and growth in this material.

\section{References:}

1. Suorsa, I., Performance and modeling of magnetic shape memory actuators and sensors, Doctoral dissertation, TKK Dissertations 4, Espoo ISBN 951-22-7644-5, (2005).

2. Söderberg, O., Sozinov, A., Ge, Y., Hannula, S.P., Lindroos, V.K., Handbook of Magnetic Materials, vol 16, ed. J Buschow, Elsevier, pp. 1-39, (2006).

3. Marioni, M.A, O'Handley, R.C., Allen, S.M., Pulsed magnetic field-induced actuation of Ni-Mn-Ga single crystals, Applied Physics Letters, 83, pp. 3966-3968, (2003).

4. Müllner, P., Chernenko, V.A., Kostorz, G., Large magnetic-field-induced deformation and magnetomechanical fatigue of ferromagnetic Ni-Mn-Ga martensites, Materials Science and Engineering A, 387-389, pp. 965-968, (2004).

5. Soroka, A., Master's thesis for the degree of Master of Science in Technology, Aalto University School of Science and Technology, 28th of May, (2010).

6. Straka, L., Heczko, O., Magnetic anisotropy in Ni-Mn-Ga martensites, Journal of Applied Physics 93, pp. 8636-8638, (2003).

7. Straka, L., Magnetic and Magneto-Mechanical Properties of Ni-Mn-Ga Magnetic Shape Memory Alloys, Doctoral dissertation, TKK Dissertations 78, ISBN 978-951-22-8819-9, (2007).

8. Aaltioa, I., Söderberg, O., Gec, Y., Hannula, S.P., Long-term cyclic loading of 10M Ni-Mn-Ga alloys, Materials Science Forum Vol.684, pp 201-212, (2011).

9. Vuherer, T., Godina, A., Burzic, A., Gliha, A., Fatigue Crack Initiation from Microstructurally Small Vickers Indentations, METABK 46 (4), pp. 237-243, (2007).

10. Nwoji, C.U., Onah, H.N., Mama, B.O., Solution of the Boussinesq Problem of Half Space using Green and Zerna Displacement Potential Function Method, Bund. 11, Vol. 22, (2017). 